\title{
Study on the Clinical Efficacy and Safety of Endostar Combined with Pemetrexed and Cisplatin in the Treatment of Lung Adenocarcinoma
}

Yanbing Wang

Pneumology Department, Taixing People’s Hospital, Taixing 225400, Jiangsu Province, China

\begin{abstract}
Objective: To analyze and study the efficacy and safety of Endu combined with pemetrexed and cisplatin in the clinical treatment of lung adenocarcinoma. Methods: From August 2016 to September 2020, 32 patients with lung adenocarcinoma who were treated in our hospital were selected for group trials. According to their specific treatment plan, the patients were divided into control group and experimental group, with 16 cases in each group. The control group was treated with pemetrexed and cisplatin, and the experimental group was treated with Endostar combined with the treatment received by the control group. The clinical efficacy and safety of the two regimens were assessed by comparing the changes in symptoms and the incidence of adverse reactions between the two groups of patients after treatment. Results: The disease control rate of the experimental group was significantly higher than that of the control group, and there was no significant difference in the incidence of adverse reactions between the two groups. Conclusions: From the experimental results, we found that the treatment of patients with lung adenocarcinoma by Endostar combined with pemetrexed and cisplatin can effectively improve the treatment efficacy without increasing adverse reactions and therefore relevant chemotherapy regimens can be considered for wider clinical applications.
\end{abstract}

Keywords: Endostar; Pemetrexed; Cisplatin; Lung adenocarcinoma; Clinical efficact; Safety

Publication date: November, 2020

Publication online: 30 November, 2020
"Corresponding author: Yanbing Wang, wyb3231923 (a)126.com

Lung adenocarcinoma is a type of non-small cell lung cancer, and it is a relatively common type of lung cancer. The cause of this disease is not fully known currently. Current clinical studies believe that the main factors affecting the incidence of lung adenocarcinoma include smoking, poor living environment, severe chemical dust pollution, malnutrition, chronic lung disease, and etc. Most lung adenocarcinomas originate from the bronchial mucosa, and some patients have lesions originating from the large bronchial mucous glands. The early clinical symptoms of lung adenocarcinoma are relatively insignificant, and patients may have no symptoms of discomfort at all. As the disease gradually progresses, patients begin to show typical respiratory disease symptoms such as coughing, hemoptysis, and dyspnea. By then, many patients are already in the mid-late or late stage of the disease upon diagnosis. Due to the relatively rapid progression of lung adenocarcinoma, the length of the patient's entire treatment cycle cannot be determined. Judging from the current clinical treatment measures for lung adenocarcinoma, radiotherapy, chemotherapy and surgical treatment are still the main treatment modalities. With the continuous deepening of related research, immunotherapy is being gradually applied in clinical work. Among various treatment modalities, chemotherapy as a systemic therapy is very crucial for patients with lung adenocarcinoma, especially for patients with advanced lung adenocarcinoma due to remote metastasis and large tumor invasion. At this stage, the patient's body is relatively poor, so 
most patients with advanced lung adenocarcinoma have lost the opportunity for surgical treatment and chemotherapy will be the main treatment modality. The growth inhibition and direct killing of tumor cells by chemotherapeutics can delay the disease progresses to the greatest extent and improves the patient's survival rate. Pemetrexed is a commonly used drug in lung adenocarcinoma chemotherapy. It is often used clinically in conjuction with cisplatin. Endostar is currently an important drug in the chemotherapy treatment of patients with non-small cell lung cancer. In order to analyze the clinical effect and safety of Endostar combined with pemetrexed and cisplatin in the treatment of lung adenocarcinoma, this paper will conduct a study on two groups of patients with different therapeutic regimens.

\section{Background and Methods}

\subsection{General Background of Patients}

The 32 patients included in the experiment were all lung adenocarcinoma patients who were treated in our hospital between August 2016 and September 2019. Among them, 16 patients were in the control group, where 11 were male and 5 were female. The age range is $49-78$ years old, and the average age is $(58.1 \pm 3.8)$ years old. Among the 16 patients in the experimental group, 10 were male and 6 were female. The age range of the patients was 48-77 years old, with an average age of (57.9 \pm 3.9$)$ years old. Patient admission criteria were: (1)All patients were diagnosed as lung adenocarcinoma by pathology and cytology. (2)The patient's expected survival period is greater than 3 months. (3) When the patient was admitted to the hospital, there was no abnormality in liver and kidney function. (4)The patient can complete the corresponding treatment plan. Exclusion criteria: (1)Concurrent serious infection. (2)Mental disorders. (3)There are other serious basic diseases. All patients and their family members understood and agreed to the corresponding treatment plan, and related experiments were reported to the ethics committee and had been approved. The general information of the patients was not significantly different, which met the requirements for comparison between groups $(P>0.05)$.

\subsection{Methods}

All patients received folic acid $(400 \mu \mathrm{g} / \mathrm{d})$ orally before chemotherapy and continued to take them until 3 weeks after the end of chemotherapy. Vitamin B12 injection was given before chemotherapy to build better body tolerance. Patients in the control group were treated with pemetrexed and cisplatin. The specific treatment scheme is as follows: Drug selection: pemetrexed (specification: $200 \mathrm{mg}$ ), pemetrexed $500 \mathrm{mg} / \mathrm{m}^{2}$. Cisplatin (specification $10 \mathrm{mg}$ ), cisplatin $25 \mathrm{mg} / \mathrm{m}^{2[1]}$.

The experimental group was treated with additional Endostar (recombinant human endostatin) based on the treatment scheme of the control group. Endostar (specification $15 \mathrm{mg}$ ) and Endostar 210 mg were continuously pumped for 7 days with an intravenous pump. Dexamethasone was taken orally on the day before and on the day of chemotherapy, and dexamethasone was also taken the day after chemotherapy at a dose of $4.5 \mathrm{mg} / \mathrm{session}$. During chemotherapy, symptomatic treatment was given according to the patient's adverse reactions, including ondansetron, Shengbaixin and etc to enhance the effect of chemotherapy and to reduce the incidence of adverse reactions.

\subsection{Assessment Criteria}

According to the assessment criteria of solid tumors, the short-term treatment efficiency is classified into four levels, namely complete remission, partial remission, stable and progress. The assessment content includes short-term treatment efficiency and disease control rate. The specific conditions are as follows. Complete remission: patients have significantly improved clinical symptoms. After imaging examination, the tumor volume was significantly reduced, the patient did not see further expansion of the lesion area, the patient's quality of life was significantly improved, and the treatment effect was satisfactory ${ }^{[2]}$. Partial remission: The patient's clinical symptoms have improved to a certain extent. After imaging examination, it can be seen that the tumor volume has been reduced to a certain extent, the patient's lesion area has not expanded further, the quality of life has been improved, and the treatment effect is relatively satisfactory. Stable: The patient's clinical symptoms did not change significantly after the medication, the tumor volume did not change significantly under imaging examination, the patient's quality of life did not change significantly compared to before the medication, and the tumor development was 
inhibited. Progress: The patient's clinical symptoms further aggravated. Through imaging observations, it was found that the patient's tumor volume further increased, the scope of lesion invasion also further increased, the patient's quality of life decreased, and the treatment effect was poor. Short-term treatment efficiency $=$ proportion of complete remission + proportion of partial remission, disease control rate $=$ proportion of complete remission + proportion of partial remission + proportion of stable remission ${ }^{[3]}$.

During the course of treatment, the incidence of adverse reactions of the two groups of patients was recorded. Adverse reactions include gastrointestinal reactions (nausea, vomiting, etc.), hematological reactions (decrease in white blood cells, hemoglobin, and thrombocytopenia, etc.), and neurological reactions (hyesthesia, numbness of limbs, etc.), liver and kidney function damage (abnormal liver function, abnormal kidney function). The safety of the two chemotherapy regimens was assessed based on the incidence of adverse reactions ${ }^{[4]}$.

\section{Results}

The short-term treatment efficiency and disease control rate of the two groups of patients are as follows. Among the 16 patients in the control group, 2 patients have complete remission $(12.50 \%), 3$ patients have partial remission (18.75\%), 4 patients are stable $(25.00 \%)$, and 7 patients had progressed. $(43.75 \%)$ The short-term treatment efficiency was $31.25 \%$ and the disease control rate was $56.25 \%$. Among the 16 patients in the experimental group, 4 patients had complete remission $(25.00 \%), 4$ patients had partial remission $(25.00 \%), 7$ patients were stable $(43.75 \%)$, and 1 patient had progressed $(6.25 \%)$. The short-term treatment efficiency is $50.00 \%$, the disease control rate is $93.75 \%$, and the short-term treatment efficiency $\chi^{2}=1.1660, P>0.05$. Disease control rate $\chi^{2}=4.6584, P<0.05$. See Table 1 for details.

Table 1. The Short-term Treatment Efficacy and Disease Control Rate of the Two Groups of Patients [ $n \%]$

\begin{tabular}{ccccccc}
\hline Group & $\begin{array}{c}\text { Complete } \\
\text { Remission }\end{array}$ & $\begin{array}{c}\text { Partial } \\
\text { Remission }\end{array}$ & Stable & Progress & $\begin{array}{c}\text { Short-term treatment } \\
\text { efficiency }\end{array}$ & $\begin{array}{c}\text { Disease } \\
\text { Control Rate }\end{array}$ \\
\hline Experimental $(n=16)$ & $4(25.00)$ & $4(25.00)$ & $7(43.75)$ & $1(6.25)$ & 31.25 & 56.25 \\
Control $(n=16)$ & $2(12.50)$ & $3(18.75)$ & $4(25.00)$ & $7(43.75)$ & 50.00 & 93.75 \\
$\chi^{2}$ & - & & & & 1.1660 & 4.6584 \\
$\mathrm{P}$ & - & & & & $>0.05$ & $<0.05$ \\
\hline
\end{tabular}

The incidence of adverse reactions in the two groups of patients is as follows: among the 16 patients in the control group, 5 patients $(31.25 \%)$ had gastrointestinal adverse reactions, and 4 patients $(25.00 \%)$ had hematological adverse reactions, 1 patient $(6.25 \%)$ had neurological adverse reactions, and 4 patients $(25.00 \%)$ had liver and kidney damage.
Among the 16 patients in the experimental group, 6 patients $(37.50 \%)$ had gastrointestinal adverse reactions, 4 patients $(25.00 \%)$ had hematological adverse reactions, 2 patients $(12.50 \%)$ had neurological adverse reactions, and 4 patients $(25.00 \%)$ had liver and kidney damage (25.00\%). See Table 2 for details.

Table 2. The Incidence of Adverse Reactions in the Two Groups of Patients [ $n \%]$

\begin{tabular}{ccccc}
\hline Group & $\begin{array}{c}\text { Gastrointestinal Adverse } \\
\text { Reaction }\end{array}$ & $\begin{array}{c}\text { Hematological Adverse } \\
\text { Reactions }\end{array}$ & $\begin{array}{c}\text { Neurological Adverse } \\
\text { Reactions }\end{array}$ & $\begin{array}{c}\text { Liver and Kidney } \\
\text { Functional Damage }\end{array}$ \\
\hline Experimental $(n=16)$ & $6(37.5)$ & $4(25.00)$ & $2(12.50)$ & $4(25.00)$ \\
Control $(n=16)$ & $5(31.25)$ & $4(25.00)$ & $1(6.25)$ & $4(25.00)$ \\
$P$ & $>0.05$ & $>0.05$ & $>0.05$ & $>0.05$ \\
\hline
\end{tabular}

\section{Discussions}

Lung adenocarcinoma is the most common type of lung cancer. Judging from the incidence of lung cancer in China during recent years, lung cancer poses a great threat to the health of Chinese people. Being the malignant tumor with the highest incidence rate and the largest number of deaths globally, lung cancer is rightfully the 'killer' of human health. Being the most common type, lung adenocarcinoma accounts for about $35 \%$ of all patients and its early clinical symptoms are not obvious. A considerable number of patients are already quite serious after the corresponding clinical symptoms appear. For patients with lung adenocarcinoma, many of them already had their lungs severely invaded by the tumor as 
diagnosis is usually made only in the middle or even late stage of the disease, where distant metastasis had occur. Therefore chemotherapy has always been the main treatment modality for patients with lung adenocarcinoma. Endostar is a new type of tumor treatment drug, and it is also a common drug used in the chemotherapy of non-small cell lung cancer currently. It prevents tumor angiogenesis by inhibiting vascular endothelial cells, thereby inhibiting tumor growth. Part of the metabolic functions of tumor cells depends on folic acid during growth. As a pyrrolidine based antifolate, pemetrexed can effectively affect folate-dependent cell metabolism and it is used to achieve the destruction of tumor cells by inhibiting tumor cell metabolism. Pemetrexed is commonly used in combination with cisplatin ${ }^{[5]}$. This paper studied the clinical treatment efficiency and safety of the combination of Endostar, pemetrexed and cisplatin in patients with lung adenocarcinoma through comparative analysis. The data showed that the experimental group that received Endostar had a higher disease control rate. In terms of incidence rate of adverse reactions, the use of Endostar based on conventional chemotherapy will not increase safety risks.

\section{References}

[1] Tian XZ, Shi MH, Gong GW, et al. Evaluation of the efficacy and safety of pemetrexed and bevacizumab in the treatment of elderly patients with lung adenocarcinoma $[\mathrm{J}]$. Chinese Journal of Clinical Healthcare, 2020, 23(4): 510-513.

[2] Chang LL. Clinical effect analysis of bevacizumab combined with pemetrexed in the treatment of lung adenocarcinoma [J]. Health Horizon, 2020(20): 71

[3] Mei LW. Study on the effect of combination of pemetrexed, oxaliplatin and cisplatin in the treatment of advanced lung adenocarcinoma [J]. Health Required, 2020(25): 42-43.

[4] Jun FL, Hong CZ, Ying QL, et al. Clinical effect of lowdosage apatinib combined with pemetrexed and carboplatin in the treatment of advanced lung adenocarcinoma $[\mathrm{J}]$. Clinical Research and Practice, 2020, 5(24): 13-15.

[5] Yu WC, Xu W. Observation on the efficacy and safety of concurrent chest radiotherapy of pemetrexed and etoposide combined with cisplatin in the treatment of locally advanced lung adenocarcinoma[J]. Guizhou Medical Journal, 2020, 44(7): 1089-1090. 